\title{
Unconventional reconciliation path for quantum mechanics and general relativity
}

Samuel Yuguru ( $\nabla$ samuel.yuguru@upng.ac.pg)

University of Papua New Guinea https://orcid.org/0000-0001-6312-4033

\section{Research Article}

Keywords: monopole, 4D space-time, quantum mechanics, general relativity, multiverse

Posted Date: July 7th, 2021

DOI: https://doi.org/10.21203/rs.3.rs-63303/v9

License: (9) This work is licensed under a Creative Commons Attribution 4.0 International License. Read Full License 


\title{
Unconventional reconciliation path for quantum mechanics and general relativity
}

\author{
Samuel. P. Yuguru ${ }^{1}$
}

1. Chemistry Department, School of Natural and Physical Sciences, University of Papua New Guinea, P. O. Box 320, Waigani Campus, National Capital District 134, Papua New Guinea, Tel. : +675 326 7102; Fax. : +675 326 0369; Email address: samuel.yuguru@upng.ac.pg.

\section{Abstract}

Physics in general is successfully governed by quantum mechanics at the microscale and principles of relativity at the macroscale. Any attempts to unify them using conventional methods have somewhat remained elusive for nearly a century up to the present stage. Here in this study, a classical gedanken experiment of electron-wave diffraction of a single slit is intuitively examined for its quantized states. A unidirectional monopole (MP) field as quanta of the electric field is pictorially conceptualized into 4D space-time. Its application towards quantum mechanics and general relativity in accordance with existing knowledge in physics paves an alternative path towards their reconciliation process. This assumes a multiverse at a hierarchy of scales with Einstein's gravity defined to be of circular acceleration in time reversal mode within an MP field precessing into forward time for a rotating body into space. Such descriptions also provide an approximate intuitive tool to examine physics in general from alternative perspectives using conventional methods and this warrants further investigations.

Keywords: monopole, 4D space-time, quantum mechanics, general relativity, multiverse 


\subsection{Introduction}

Since the late 1800 s to early1900s, knowledge acquired in increments for the microscale with the advancement of proper experimentations has come to successfully form a fundamental theory of the atomic state known today as quantum mechanics. An unexhausted list of scientists that contributed to the development of the theory during this period of time can be found in any common textbook. It was only during the 1920s that the theory was fully construed in what came to be widely known as Copenhagen interpretation, a phrase attributed to Niels Bohr and Werner Heisenberg [1]. The interpretation relates to the fundamental level, where both particle-like and wave-like characters' exhibit wave-particle duality. In addition, neutral to charged particle-like properties possess superposition states of spins in probabilistic distributions. Both of these quantum features appear somewhat counterintuitive to everyday notions offered by classical mechanics. In order to account for each of the weirdness of quantum mechanics, other alternative versions like Everett's many-worlds interpretations, quantum Bayesianism, De Broglie-Bohm theory among a few others exist. But these are not as popular as the Copenhagen interpretation based on experimental outcomes conducted so far.

Coinciding with the development of quantum mechanics in which Albert Einstein also played a key role in defining the particle property of light waves as photons [2], he further formulated his two prominent relativistic theories [3]. Special relativity accounts for constant lightspeed of wave-particle duality in a vacuum for all accelerating inertia frames of reference. Likewise, it denotes mass-energy equivalence for matter into space in one of the most famous equations in physics, $E=m c^{2}$. Their combination with quantum mechanics paved the path for 
the emergence of the quantum field theory (QFT) and this provides the link between the microscale and the macroscale or the classical level (Fig. 1). The process first began in the early

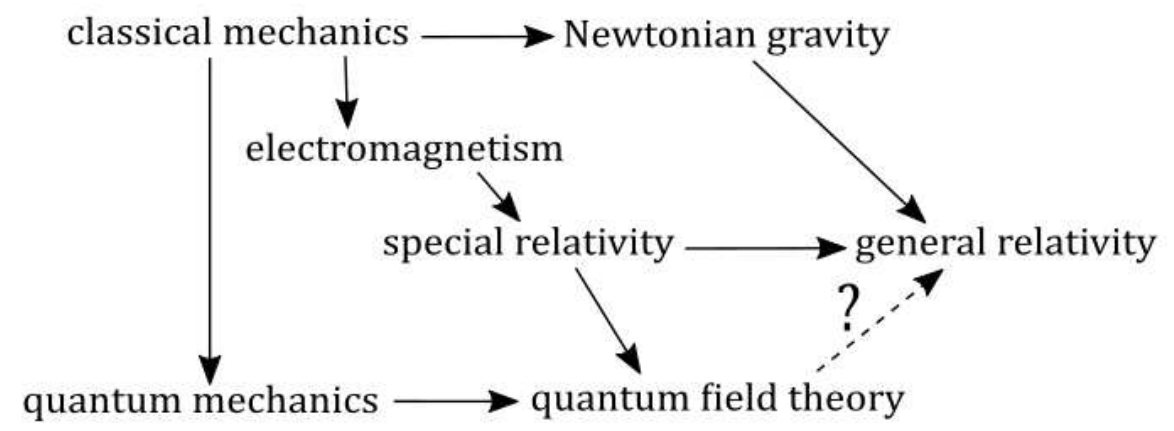

Figure 1. A simplified flow diagram demonstrating the gap in conventional methods towards the unification process of quantum mechanics and general relativity.

1930s with Paul Dirac's initial proposition of the existence of antimatter [4] in the form, $\mathrm{e}^{+} \mathrm{e}^{-} \rightarrow$ $2 \gamma$, where an electron annihilates with its antimatter to produce two gamma rays. Such a prospect was further promoted by Richard Feynman [5] in the late 1940s using path integral diagrams, where particles couple to their antimatter to generate different force types like electromagnetism, weak and strong nuclear forces. Except for gravity, the combination of the particles and forces in accordance with experimental findings was successfully completed over the next few decades through the development of the QFT known as the Standard Model (SM) [6]. The SM theory of elementary particles employs very complex mathematical formulations to account for a plethora of particle types observed in high energy experiments. Its final prediction successfully culminated in the discovery of the Higgs boson in 2012 [7], where its existence was predicted in the mid-1960s [6]. Depending on their spins, charges and masses, the fundamental particles in the SM are categorized either as fermion or boson types. The bosons are of whole integer spin $(0$ 
to \pm 1 ) and these are considered to have been generated from the coupling of fermions and their antimatters (i.e., particles of $\pm 1 / 2$ spin to other fractional spin types). The bosons mediate the main field forces of electromagnetism, weak and strong nuclear forces. A different boson type of spin 2 (graviton) is assumed to mediate gravitational force but this has not been detected yet in experiments [8]. Its eventual discovery and incorporation into an extended SM is expected to provide the possible unification path of quantum mechanics and general relativity at the fundamental level [9]. However, one major constraint to such endeavor is that gravity is envisioned to weakly manifest at the Planck's length of approximately $10 \mathrm{~cm}^{-34}$, a scale of very high energies not accessible yet in current experimental undertakings [10].

At the macroscale, Einstein's other landmark theory of general relativity successfully equates Newtonian gravity to acceleration with respect to an inertia frame of reference in what came to be known as the equivalence principle [11]. For an observer in an accelerated frame of reference, gravity is known to curve space-time fabric with the integration of special relativity (Fig. 1).

Overtime, experimental findings and new theories have evolved to affirm the accepted common knowledge in both quantum mechanics and general relativity as the two pillars of physics at two extreme scales. To date, any attempts to unify them using conventional methods such as the QFT applications of the SM or quantum gravity remained fairly constrained since earlier attempts by Einstein in the 1930s [12]. One major hurdle to such quests is that these methods are exclusively grounded on abstract mathematical tools that are largely driven by empirical data or vice versa. Hence, for this study, a non-mathematical approach of a conceptualized form into four-dimensional (4D) space-time or 3D space plus 1D time is 
considered towards the reconciliation process of quantum mechanics and general relativity. This is briefly outlined below.

A classical Einstein's gedanken experiment of electron-wave diffraction of a single slit [13] is pictorially examined for its quantized states. Condensed electric field, $\mathbf{E}$ of the wave diffraction generates a unidirectional monopole field (UMF) as its quanta [4]. The UMF consists of a monopole pair (MP) field of an elliptic shape in precession mode into forward time. Its dissection linearly along inertia frames of magnetic field, $\mathbf{B}$ generates circular Bohr orbits (BOs) in degeneracy. These insinuate quantized states into extra dimensions along orbital paths within the MP field. Precession or acceleration of the orbital of a circular motion is of time reversal mode to the MP field and this attributed to gravity in accordance with the equivalence. This generates the emergence of an MP model into 4D space-time of a rosette shape. For the microscale, output of Planck's radiation breaks the time reversal symmetry imposed by gravity consistent with the $2^{\text {nd }}$ law of thermodynamics and this somewhat mime's the curved geodesic motion at the macroscale for a multiverse of MP models at a hierarchy of scales. Interaction with an external light path travelling in a straight line sustains both superposition states of quantized fields or particle-like properties and gravitational time dilation along the BOs. In this way, the structural frame of the MP model remains hidden to observation at lightspeed. Its application to physics in general is able to integrate well existing knowledge for both the microscale and the macroscale into proper perspectives. Such outcome provides a tangible unification path for quantum mechanics and general relativity. In addition, the proposed model offers a dynamic intuitive tool that can be applied to explore physics in general from alternative perspectives using conventional methods, perhaps in incremental steps and this warrants further investigations. 


\section{0 Conceptualization process of an MP model}

The conceptualization path of the MP model is attempted from a classical gedanken experiment of electron-wave diffraction of a single slit. First, the process is devised using pictorial demonstrations. Second, the model is validated by applying a generalized renormalization process based on common knowledge in physics. Third, its notable limitations are examined with suggestions offered on how these can be intuitively accommodated into a probable state of 4D space-time. The final outcome offers a dynamic intuitive tool and this is applied to explore physics in general from the microscale to the macroscale wherever applicable.

\subsection{A pictorial demonstration}

An observer at a slit sees ripples of spherical waves receding into forward time for an emitting electron source (Fig. 2a). The electron possesses both isospin, $I_{z}$ and energy-momentum, $\Phi$. On expansion, the former is projected as arrow of time, $\vec{I}$ in asymmetry of unidirectional and this is dissected perpendicularly along inertia frames of $\mathbf{B}$ into straight paths. The condensed boundary of $\mathbf{E}$ encompasses $\vec{I}$ at a minimal energy level of Planck's constant, $h$ (i.e., $6.626 \times 10^{-34}$ joules per second). This insinuates a UMF of multiple MP fields (Fig. 2b). The emergence of orbital structures within an MP field somewhat mimes a UMF background in thermal equilibrium (Fig. $2 \mathrm{c})$. The orbitals are quantized linearly along inertia frames of BOs defined by $\Phi$. These are of degenerate states into extra dimensions in time dilation, $I_{z \|}$ due to gravity with respect to $\vec{I}$ (Fig. 2d). Precession of the MP field into forward time of a circular motion is equally balanced out by 
a

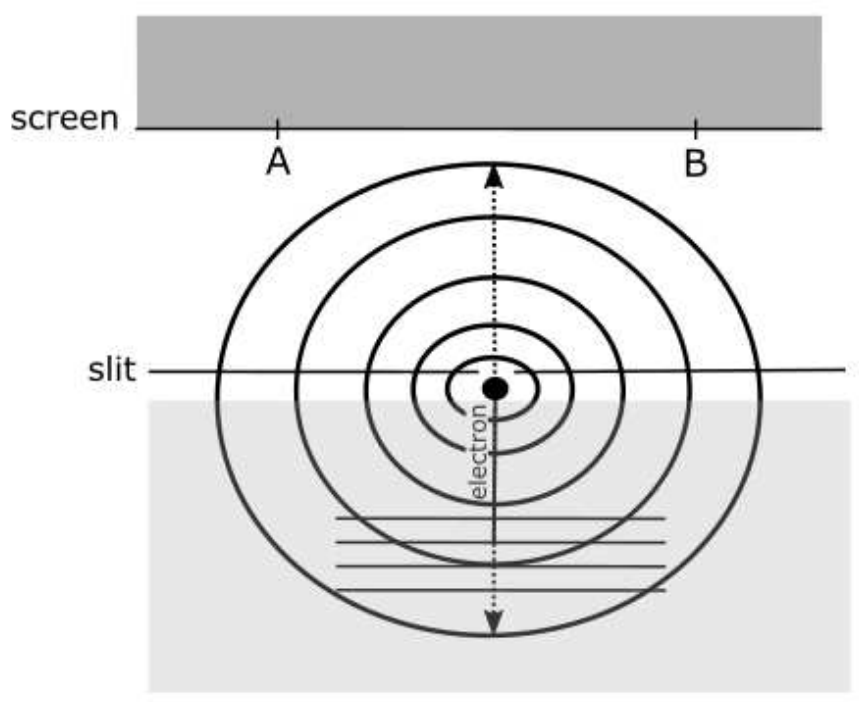

C

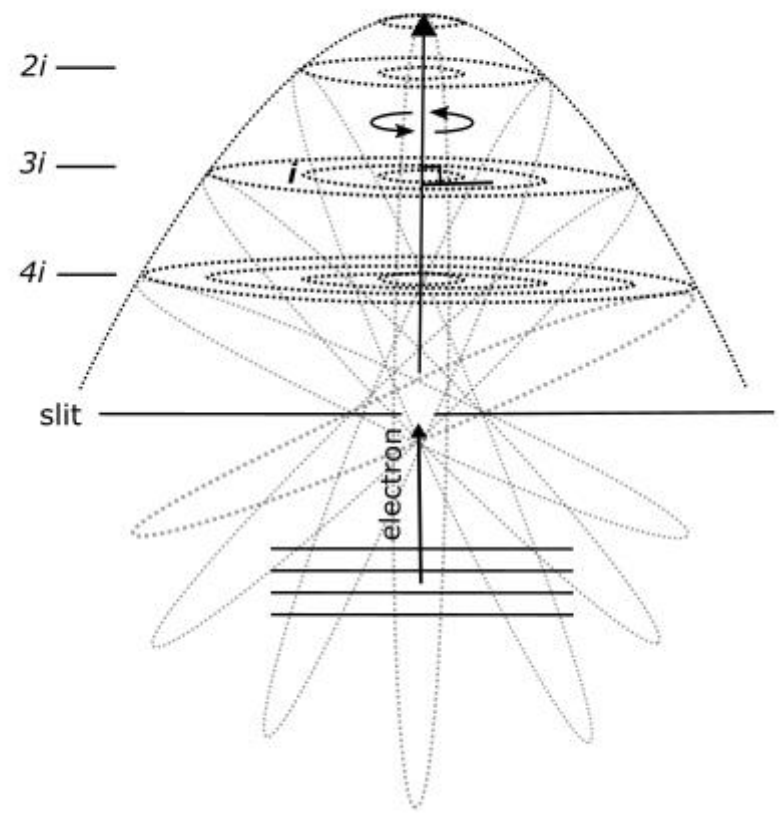

b

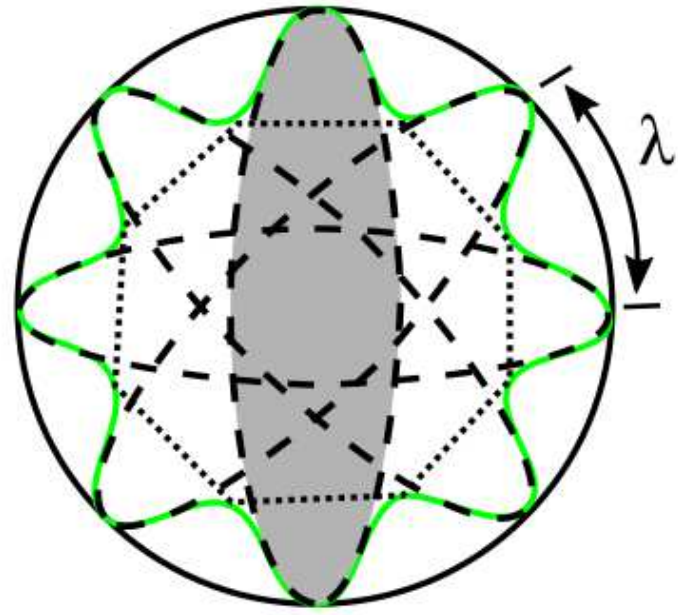

d

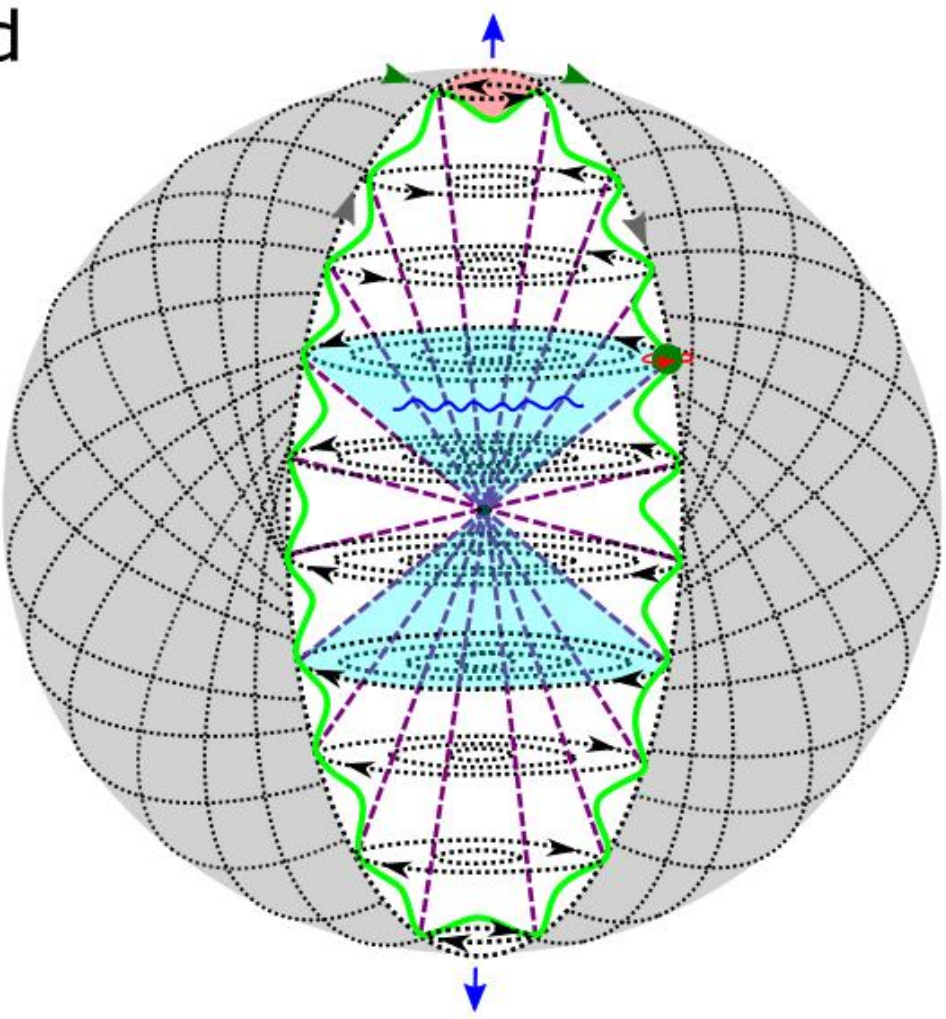

Figure 2. A step-by-step conceptualization path of the MP model. (a) Expansion of electron-wave diffraction from an electron source in a single slit setup towards detectors A and B. (b) $\vec{I}$ in asymmetry is incorporated by the UMF (green loop) within the surface of a sphere and this includes the emergence of 
an MP field (gray area) into 3D space. An octet shape of inertia frames is enclosed within a circular $\mathbf{E}$ and coherence is epitomized by the wavelength, $\lambda$ for conservationism. (c) Emergence of orbital structures within the MP mimes the UMF background in thermal equilibrium. These are quantized along the $\mathbf{B}$ of BOs in degeneracy and into extra dimensions, $n_{i}$. (d) The MP field is transformed into 4D space-time of unidirectional of a rosette shape in flat space. The orbital paths (pink dotted lines) of a point particle (green circle) such as an electron are quantized. Miniature wave function, $\Psi$ (blue wavy curve) of gravitational type traverses the orbital paths. The electron transition between two orbitals from point $x \rightarrow$ $\hat{x}$ (pink area) equates to $\pm h$ of Planck's radiation for the MP field (white area). This offers infinitesimal steps for precessing MP field of a clock face (green arrows) and in turn breaks time reversal symmetry imposed by gravity. Such a process generates time dilation towards the determination of the $\pm \frac{1}{2}$ magnetic spin $\left(m_{s}\right)$ (navy colored cones) in superposition states along the BOs. Planck's radiation sustains $\vec{I}$ (blue arrows) in asymmetry in accordance with $2^{\text {nd }}$ law of thermodynamics, where conservation is assumed for the microscale (green wavy loop). All these descriptions allude to the dynamics of an MP model of a spherical electron cloud model (gray area) into 4D space-time in flat space.

the orbital's precessions into time reversal mode. The former mimics a clock face, while the latter is interpreted to be of Einstein's gravity in accordance with the equivalence principle. In this way, both fields and particle-like properties in superposition states are applicable at the inertia frames or $n$-levels of BOs into extra dimensions. Increase in the dimensions towards the nucleus somehow coincides with the complexities of the orbital types, i.e., $s \rightarrow p \rightarrow d \rightarrow f$ and so forth. The mass-energy equivalence of $E=m c^{2}$ at a defined $\lambda$ of the electromagnetic radiation or light wave is sustained along the BOs (Fig. 2d). In the absence of any external interaction, the Planck's radiative process breaks time reversal symmetry imposed by gravity in accordance with the application of $2^{\text {nd }}$ law of thermodynamics, i.e., $E=n h c / \lambda$. Singularity is defined by $i \hbar$ at a minimal energy level of Planck's scale at the point-boundary of the MP model (Fig. 2d). This 
supposes that in a double slit experimentation, the Planck's radiation is evenly distributed within the electron-wave diffraction patterns. All these descriptions allude to an MP model that somewhat resembles an electron cloud model into 4D space-time. This is applicable to either one-electron atom such as hydrogen for an MP field or multielectron atoms for multiple MP fields. The electrons probability distributions along the orbital paths into extra dimensions of BOs are attained in accordance with Pauli exclusion principle. In the subsequent subsections, the model's compatibility to a renormalization process and limitations are explored.

\subsection{A generalized renormalization process}

The conceptualization path of the MP model can be conceived in accordance with common knowledge in physics. Commencing from the electron source towards the generation of spacetime, the process is conceived by a triple integral in the following manner

$$
\int_{-\infty}^{\infty} d I_{z} d \phi \rightarrow \int_{-\infty}^{\infty} \int_{0}^{\pi} n I_{z \|} d \phi d \Omega \rightarrow \int_{-\infty}^{\infty} \int_{0}^{\pi} \int_{0}^{2 \pi} n I_{z \|} d \phi d \Omega d \theta \quad .
$$

Perpetual rotation of the UMF insinuates 4D space-time and this is defined by $h$ in infinitesimal steps at the microscale due to intermittent precession of the MP field defined by $2 \pi$. Gravitational time dilation, $I_{z||}$ is attained along the quantized states of BOs on the orbital paths at the $n$-levels.

The BOs are of degenerate states and these appear perpendicular to the principal axis, $\vec{I}$ of the MP field in asymmetry (Fig. 2d). Non-relativistic position of a particle along BO into 3D space at a point into space-time $(x, t)$ is defined by the spherical polar coordinates $(\Omega, \Phi, \theta)$. The 
symbol $\Omega$ equates to the number of configurations of the orbital's precessions within the MP field in a circular motion. $\Phi$ is the stress-energy momentum of BO from competing forces of gravity and precessing MP field into forward time. $\theta$ is the angle between $I_{z \|}$ and $\vec{I}$ with respect to singularity at the electron source. The designation of $\Phi$ to the degenerate BOs into in $N$ dimensions of Hilbert space somewhat mimics a Higgs field (Fig. 2b and c). Hence, a plethora of particle types are envisioned depending on the applied energy levels. Any point particle in situ of the MP model above Planck's energy assumes $\pm \frac{1}{2} m_{s}$ in superposition states along the BOs (Fig.

2d). The normalization of Equation 1 then takes the form

$$
n I_{z \|} \int_{-\infty}^{\infty} d \Omega d \phi d \theta=1
$$

where the integral incorporates the path covered by the particle in orbit and is of time invariance for the MP field. From the first principle of the quantum $\Psi$, a particle's position in orbit is also related to the Hamilton-Jacobi relationship of the type

$$
n I_{z \|}=a b c \Omega \sin \theta \quad .
$$

The parameters $a b c$ represent the lengths of semi-principal axes of a BO (Fig. 3). The renormalization process then becomes of the generalized form

$$
\frac{a^{2}}{x^{2}}+\frac{b^{2}}{y^{2}}+\frac{c^{2}}{z^{2}} \leq 1
$$


where the volume of the ellipsoid is given by, $v=4 \pi a b c / 3$. Projection of BO into extra dimension along the $x$-axis generates quantized energy states at the $n$-levels (Fig. 3).

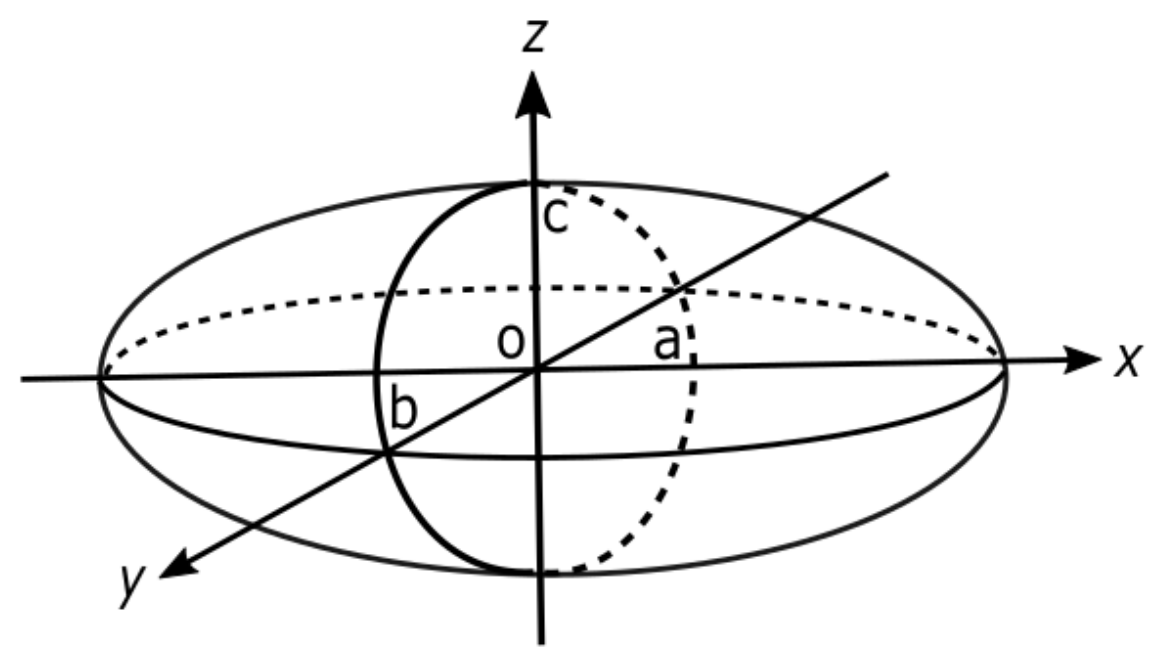

Figure 3. An MP field of an elliptic shape. The circle along the $z$-axis $/ I_{z \|}$ is comparable to a $\mathrm{BO}$, while the $x$-axis identifies $\vec{I}$ in asymmetry. In turn, the circle possesses $\mathbf{B}$, whereas the elliptic shape is of an $\mathbf{E}$.

Alternatively, Equation 3 can be expanded into the form

$$
d x d y d z=a b c \Omega^{2} \sin \theta d \Omega d \Phi d \theta
$$

where $\Omega^{2}$ depicts precessing MP field between two points and this insinuate a $\Psi$. Such a process is conceptualized later in the text. The magnitude of the $\Psi$ is dependent on the size of the object interacting with photons travelling on a straight path. For the particle transition between two orbitals from point $x \rightarrow \hat{x}$ within an MP field (Fig. 2d), this follows Born's rule in the generalized form 


$$
n I_{z \|} \int_{-\infty}^{\infty} \Psi^{*} \Psi d \tau=1
$$

where $d \tau=d x d y d z$ and the dimensions of BO defined by $n I_{z \|}$. Equation 6 holds true from the first principle, where the $\Psi$ is applicable to all constants such as an electron or quantized states of BO. Such intuition forms the basis for the physical derivation of Schrödinger's equations and this is explored later in the text. Depending on the experimental set-up, external light application at a point-boundary of the MP model is expected to generate standing waves in $2 \mathrm{D}$ space of a continuum mode (i.e., Euler's formula). This is defined by the relationship

$$
\Psi=A \sin \frac{2 \pi x}{\lambda} \quad \text { or } \quad B \cos \frac{2 \pi x}{\lambda}
$$

where $\mathrm{A}$ and $\mathrm{B}$ are constants with $\lambda$ projected along the $x$-axis into $2 \mathrm{D}$ flat space. All these descriptions offered in this section allude to the dynamics of the MP model into 4D space-time.

\subsection{Limitations}

Firstly, the most obvious question arises from what powers the precession mode of the MP model into forward time in a perpetual motion and in turn violate the laws of thermodynamics? By linking precession to $\pm h$ for the MP field into forward time invariance of $\vec{I}$ at the Planck's scale, energy is conserved and a possible ultraviolet catastrophe is avoided. Planck's radiation 
then breaks time reversal symmetry imposed by quantum gravity in accordance with the $2^{\text {nd }}$ law of thermodynamics (e.g., Fig. 2d). Perpetual rotations in one direction then offer a miniature clock that somehow mimes a large time clock since the Big Bang. Additional details are provided later towards the final section of this paper.

Secondly, suppose gravity or specifically acceleration in reversal mode increases towards the nucleus, observations of the orbital structures become more complex into forward time, such as the $\Psi$ of hydrogen atom into 3D space. Such intuition is somewhat highly speculative but this cannot be effectively addressed by the principle of Occam's razor. For example, the principle underlies conventional methods and this requires empirical data, such as from point A to point B in $2 \mathrm{D}$ to $3 \mathrm{D}$ spaces. In the process, any intricate details, intuitions or complexities between the two points in a possible 4D space-time are overlooked.

Thirdly, even to translate the large data gathered in various interrelated disciplines in physics and collate them into a proper perspective of 4D space-time remains lacking. Without any new insights offered by experiments conducted so far, the way forward for physics appears to have reached a stalemate. Likewise, the SM theory has been quite successful in integrating the known elementary particles and the three force types of nature, i.e., electromagnetism, weak and strong nuclear forces [6]. However, beyond that, it appears inadequate to account for quantum gravity, dark matter and dark energy among others in the absence of new empirical data [7].

Finally, in order to further ascertain the dynamics of the orbitals structures or the rigor of the MP model into 4D space-time (Fig. 2d), this is weighted against common knowledge in physics. In this process, physics is explored from the perspective of 4D space-time towards the minimal dimension of 2D space-time rather than the other way around as adapted by conventional methods. Currently, there are no existing studies in physics that have pursued such 
a path before in order to avoid being metaphysical. To maneuver through this hurdle, the proposed model's compatibility to a renormalization process is first established as shown in the preceding subsection. In the subsequent sections, its applicability to symmetry is conceived before examining its relevance to existing knowledge in physics for both the microscale and the macroscale wherever applicable.

\subsection{MP model versus symmetry}

Symmetry at the fundamental level is governed by the Noether theorem and this assumes energy conservation. Its application to physics requires the existence of both matter and antimatter as first proposed by Dirac [4] for the electron-positron pair. Evidences of antimatter are provided by the discovery of positron [14] and stern-gerlach experiment of $\pm \frac{1}{2} m_{s}$. Beyond that, other empirical data for the existence of supersymmetric partners or microscale black holes are still lacking $[7,15]$. While these are still being investigated into ongoing research developments, an intuitive demonstration of symmetry for $\pm \frac{1}{2} m_{s}$ offered in Fig. 2 d is expounded in Fig. 4a. By assuming a multiverse at a hierarchy of scales, the process is applied to the solar system (Fig. 4b). Because $E=m c^{2}$ and the equivalence principle are sustained along the inertia frames of BOs, the structural frames of the MP model remain hidden to observations into forward time. Once plausible reason is that the frames are time dilated by gravity. Hence, with the area of the applied light more than the atomic state, both matter and antimatter are observable depending on the instrumental set-up. At the macroscale, the reflected light rays are less than the area of the planet and thus, superposition states are not readily applicable to an external observer. Instead, 
a

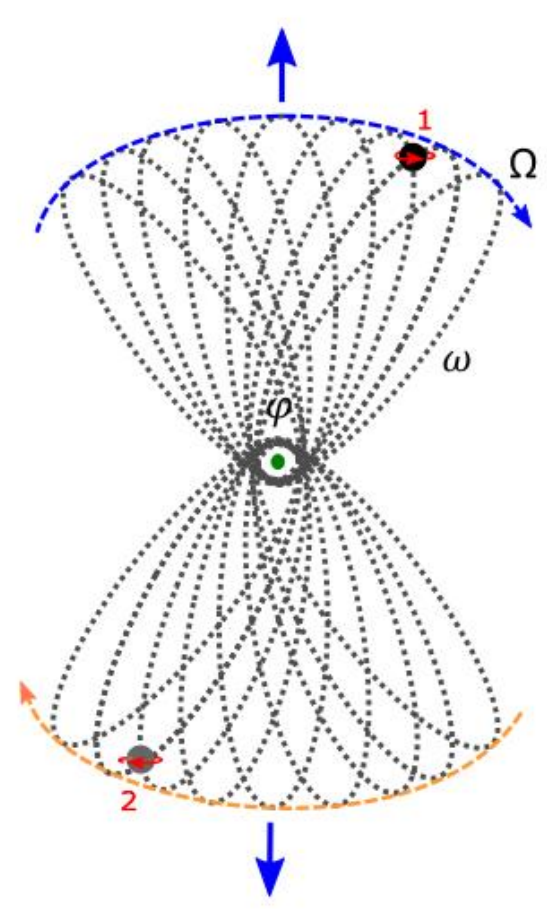

b

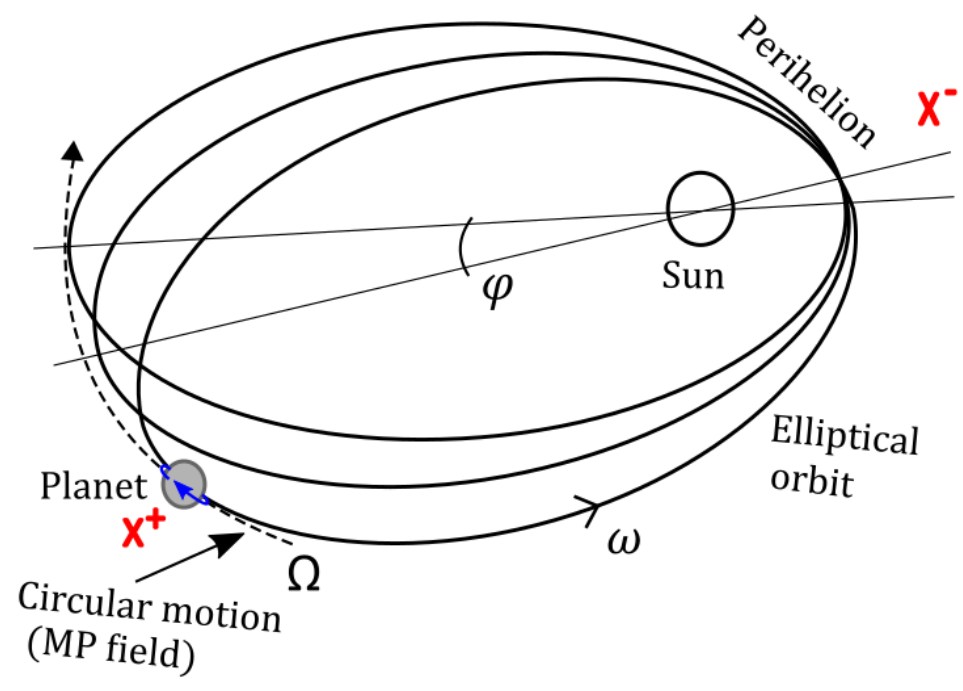

scales. (a) At the microscale, a particle extracted in an upward direction at position 1 during observation offers a $-\frac{1}{2}$ spin and vice versa in a downward direction at position 2 . The spins are respectively attained counterclockwise or against precessions of the MP field into forward time of unidirectional (blue and brown dotted curves). In this way, superposition states of $\pm \frac{1}{2} m_{s}$ are sustained into extra dimensions along BOs towards a rotating nucleus mimicking Einstein's gravity. (b) A similar process is perhaps envisioned at a higher hierarchy of scale for Mercury's orbit [16]. The orbital's shifts in its perihelion precessions are owed to gravity and this is balanced out by the MP field precessing into forward time. Only one part of the MP is accessible to an external observer unlike the microscale. $\mathrm{X}^{+}$represents matter spinning counterclockwise and $X$ - is the apparent position for the antimatter field. The latter is not drawn according to spatial distance. $\omega=$ perturbation of angular velocity and $\varphi=$ is the measure of magnetic flux of BOs into extra dimensions between two precession stages of the orbital paths.

an MP field is assumed to normalize the outward precession of the orbital paths, while perihelion precession is attributed to Einstein's gravity balancing out forward time (Fig. 4b). Such intuitions 
demonstrate how symmetry in relation to energy, magnetic momentum, angular momentum, magnetic spin and center-of-mass are sustained within the MP model into 4D space-time at a hierarchy of scales. Establishing these features offers a dynamic intuitive tool that can be applied to examine physics in general for both the microscale and macroscale from an alternative perspective and this is explored next.

\subsection{MP model versus various aspects of physics}

In this section, the model's relevance to both the classical and quantum physics is offered. Such a process is expected to integrate a number of physics themes based on experimental outcomes into proper perspective. At the moment, this is lacking from current observations and theories without any forthcoming new insights from experiments despite the advancement of instrumentation made in recent times [17].

In Fig. 5, the treatment of the MP model to various physics themes for the microscale is demonstrated. Some of these include Planck's radiation, wave function collapse, Euler's formulation, Schrödinger equation, Heisenberg uncertainty principle, Bohr's model, electromagnetism and so forth. These are briefly expounded below in bullet points.

- Wave function collapse: The presence of a particle such as an electron in orbit smoothens out the precession stages of the MP field and this insinuates the emergence of a physical $\Psi$ into $4 \mathrm{D}$ space-time. The $\Psi$ is of time dilation due to gravity and contains all the information about the system. Its intermittent transition from point $x \rightarrow \hat{x}$ between two orbital paths on a straight line is of a continuous mode for a rotating sphere. This 
somehow relates to the Hermitian operator during observations and it indicates the wave function collapse of probabilistic distributions (Fig. 5). The output signal is spiked in the

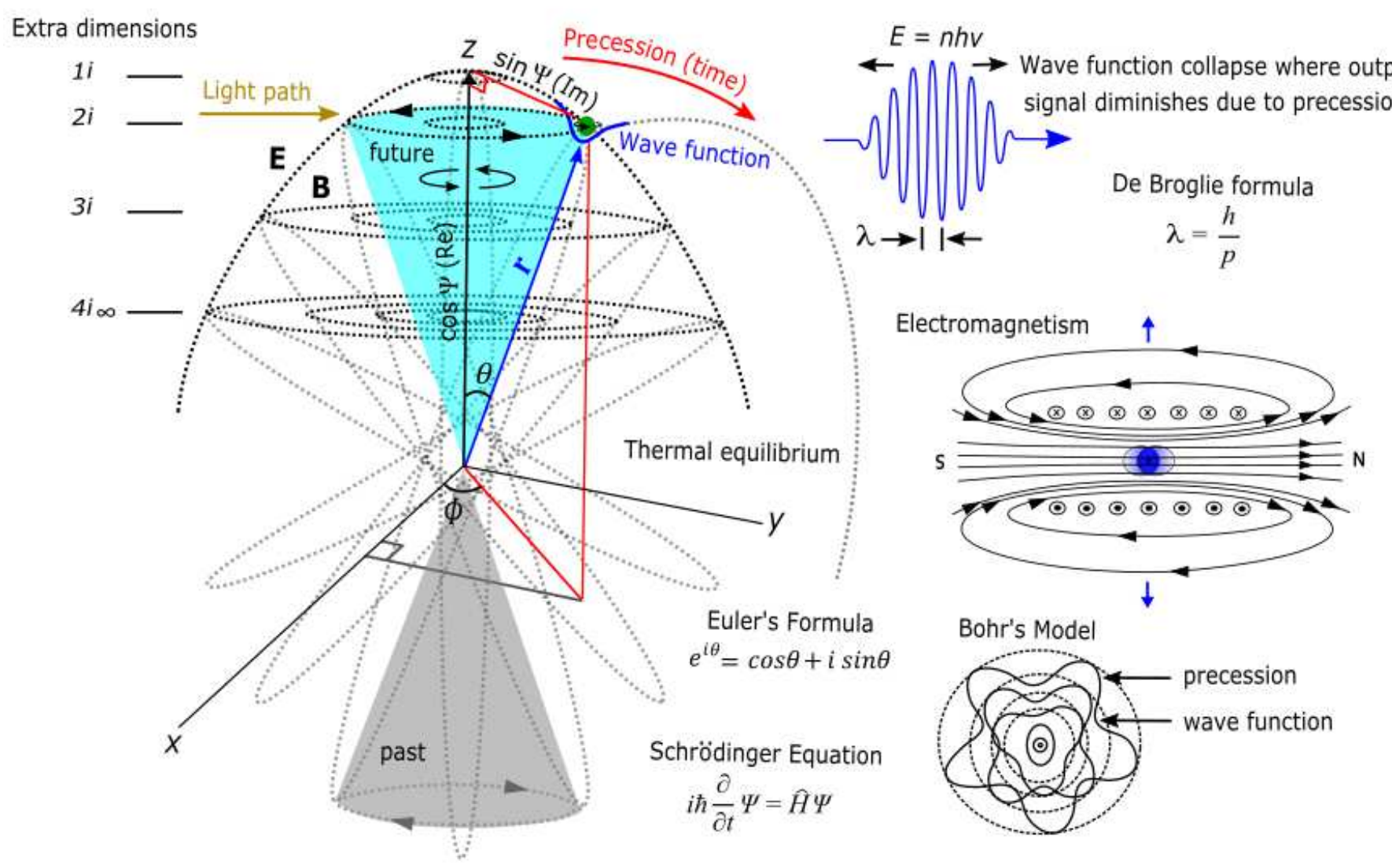

Figure 5. The application of the MP model to quantum mechanics into 4D space-time. The transition of an electron between two orbitals into space is characterized by, $x \rightarrow \hat{x}$ and this resembles $\psi \rightarrow \psi^{*}$. The imaginary (Im) part of the $\Psi$ depicts the current position of the electron before it shifts to the future or real (Re) part along $z$-axis. The precession of the orbital paths in a circular motion into extra dimensions, $2 i$ etc, (within the MP field precessing into forward time) is of time reversal mode based on Einstein's gravity. This somewhat intuitively incorporates the Euler's formula, $e^{i \pi}+1=0$, where $i=\sqrt{-1}$ is an imaginary complex number that relates to the electron's position along a distance, $r=1$ with respect to the center of the circle, $\pi$. Measurement into forward time reduces the $\psi$ into probabilistic distributions (i.e., a wave function collapse). Descriptions of the other themes illustrated here are expounded in the text. 
form, $E=n h v$. The electron's wave-particle duality is defined by the De Broglie relationship, $\lambda=h / p$. The momentum, $p=m v$ is attained along BO with $m$ equal to mass and $v$ is velocity, while in orbit. Such intuition considers the particle to be a physical entity and its probability distribution can somehow translate to the wave diffraction pattern noted in the double slit experiments, by assuming quantum tunneling effect for precessing orbital paths during expansion and this warrants further investigations.

- Schrödinger Equation: The electron's position is non-relativistic and it is described by the $\Psi_{n, l, m}$ of time dilation. It has two parts for the spherical coordinates. The radial component, $R_{n, l}(r)$ and the angular component, $Y_{l, m}(\theta, \Phi)$. Both of these features are intuitively accommodated in Fig. 5, where BO is defined by $\phi$ as mentioned earlier. It is crucial to note that observation determines the outcome of $\pm \frac{1}{2} m_{s}$ in superposition state consistent with Schrödinger's cat narrative. A level of indetermination on the outcome of the $m_{s}$ is offered by precession of the MP field into forward time against gravity in time reversal mode. Time evolution of the $\Psi$ or shift in the electron's position is of the generalized form, iћ $\partial / \partial t \Psi=\widehat{H} \Psi$ where $\widehat{H}$ is the Hamiltonian operator for the total energy. In situ of the MP model into 4D space-time, the $\Psi \rightarrow \Psi^{*}$ transition (Fig. 5) obeys Born's rule for complex probability amplitudes. All these features can be further explored using conventional theoretical applications for complex mathematical formulations.

- Heisenberg uncertainty principle: The position of the electron is described by the relationship, $\Delta x . \Delta p \geq \hbar / 2$. Based solely on Fig. 5, the uncertainty principle appears to be an inherent property of the quantum state and not as a measurement dilemma. For example, $h$ is defined by precession of the orbital within an MP field (Fig. 2d), and this somewhat offers level of indetermination on both the position, $\Delta x$ and momentum, $\Delta p$ of 
the electron. In this way, the electron's position is quantized, iћ into space. Any information transfer between the electron and the photon incorporates the quantum parameters, $n, l, m$ and $m_{s}$. The $n$-level relates to extra dimensions, $l$ is defined by $\theta, m$ is attributed to the BOs in degeneracy and $m_{s}$ in superposition states corresponds to light cones for both the past and future settings (Fig. 5). Applying such intuitions, how degenerate BOs could relate to Zeeman effect and perhaps other odd spin types for the rotating MP model against quantum gravity can be further explored.

- Bohr's model: The MP model is a literal representation of Bohr's model into 4D spacetime of a sphere. Quantization of the orbital paths within the MP field into flat space is attained along inertia frames of BOs (Fig. 2d). Because quantization epitomizes particle presence, the outgoing radiation translates to discrete energies in form, $E=n h v$ for the wave function collapse. As a consequence, the electron is not expected to spiral inwards towards the nucleus with continual radiation in accordance with the $2^{\text {nd }}$ law of thermodynamics. Thus, the past is not always equal to the future or vice versa with possible quantum decoherence from interactions with the surroundings. Such intuitions are also applicable to multielectron atoms for multiple MP fields. This assumes that increase in dimensions towards the nucleus is expected to produce complex orbital types, while sustaining Pauli exclusion principle and this warrants further investigations.

- Classical electromagnetism: Based on the conceptualization process (Fig. 2a, b, c and d), the spherical boundary of the MP model is defined by $\mathbf{E}$, while its quantization along BOs in degeneracy possess $\mathbf{B}$. With conservation sustained, a particle such as an electron in orbit identifies with a classical Maxwell point. Shifts in its position due to precession are incorporated into the relationship, $\nabla \times \mathbf{E}=-\partial \mathbf{B} / \partial \mathrm{t}$ with $\nabla$ defined by the spacing of 
BOs into extra dimensions. The precession of the orbitals is balanced out by precession of the overlaying MP field and this in turn influences the rotational of $\mathbf{B}$ along BOs. The point-boundary of the MP model is defined by $i \hbar$ as mentioned earlier. Its interaction with a light path translates to the standing waves of the electromagnetic field of Euler's formulation (e.g., Equation 7). Similarly, any external application of magnetic fields during compression of the MP model is expected to insinuate Casmir effect (Fig. 5) and this warrants further investigations.

- A probable entanglement scenario: Precession of the MP field into forward time against gravity offers a level of indetermination for the $\pm \frac{1}{2} m_{s}$ in superposition states along BOs for the $\Psi_{n, l, m}$ (Fig. 5). Light interaction with the BOs of time dilation allows for translation of 4D space-time into 2D space (i.e., wave function collapse or quantum decoherence). Linear coupling of a complementary pair of $m_{s}$ for an MP model pair is expected to produce qubits, 1,0 . In this case, photons are massless and acquire the quantum information from direct interactions with the MP models or alternatively electrons (Fig. 5). Whether such information between a complementary pair of photons at a distant can be correlated in the absence of light as a transport medium poses an interesting prospect for quantum entanglement. Similarly, whether the information gained by the photons since the Big Bang is either conserved or destroyed through quantum decoherence poses another intriguing dilemma for further considerations.

- Statistical mechanics: The MP field encloses precession stages of the orbitals in an unlimited configuration states per time and this is defined by $\Omega$ (Fig. $4 \mathrm{a}$ and b). Both the orbital and the MP field are in thermal equilibrium with the UMF (Fig. 2b). Quantization of the orbitals along BOs provides the microcanonical ensemble for the entropy, $S$ in the 
form, $S=k \operatorname{In} \Omega$. The Boltzmann constant, $k$ offers an approximate value to the distributions of both fermions and bosons along degenerate states of BOs into extra dimensions of randomness. Within the MP model, the $N$-dimensions of Hilbert space can be explored for the application of both matrix and algebraic math for both Dirac-Fermion and Bose-Einstein relationship. Extension of the baryon octet shape (Fig. 2b) towards the nucleus may accommodate decuplet diagrams for quark distribution for a multiverse at a lower hierarchy of scale. Such descriptions can be explored for the SM, where quantum excitations of the degenerate BOs at $n$-levels may account for a plethora of particle types.

- Further experimental pursuits: The design of the MP model of a gyroscope prototype resembles a rosette shape (Fig. 2b) and this can be tested experimentally into 4D spacetime. The overall rotation should mimic a clock face, while its internal orbital structures precess into time reversal mode as Einstein's gravity. An object in orbit with spin into forward time and its interaction with a light beam can be observed for a number of scenarios. 1) Lightspeed at less than the speed of the rotating gyroscope. 2) Lightspeed at almost equal to the gyroscope's speed. 3) Lightspeed at faster than the gyroscope's speed. Any successful outcomes of such undertakings is expected to ascertain some of the quantum features described above and this warrants further investigations.

\subsection{MP model versus General Relativity}

Relativistic theories in physics form the cornerstone for cosmic observations. Einstein's name is synonymous with their development and this involves more complex mathematical paths that are construed to generally comply with the experimental findings. But to somehow translate such 
information into a proper perspective of 4D space-time is still lacking. Even for QFT applications, abstract mathematical tools know no physical boundaries and the predictions of extra dimensions offered by theories such as string theories or loop quantum gravity remain unphysical and these are yet to be tested and proven in current experimental undertakings [7, 15]. With the compatibility of the MP model into 4D space-time and its wider application to both quantum and classical physics demonstrated in the preceding sections, its applicability towards relativistic theories is further explored in here. First a probable black hole scenario is assessed and this is followed by the applications of general relativity on the solar system. Other notable themes like the Big Bang and cosmic inflation are briefly explored at the end of this presentation in order to pave a new research path for their future pursuits.

\subsection{A black hole scenario}

Without any forthcoming information obtained directly from a black hole, its true nature remains concealed. Only light interaction with the surrounding matter is indirectly applied to predict the probable nature of a black hole [18]. At the moment, this is one of the most intensely researched topics that are currently being pursued by collaborations between countries of different continents. A feat never undertaken before, where satellite discs at long distances are positioned to synchronize with each other in a matter of several minutes [19]. One of the most important aspects of the black hole is that it is the place, where both classical and quantum mechanics are expected to come into the foreplay [18]. But trying to elucidate this process towards a possible unification path of quantum mechanics and general relativity offers an enigma that continues to persist today. 
In our universe, two populations of black holes are presumed to exist, each at the nucleus of every galaxy [20]. Those of stellar-mass with masses in the range of 5 to 30 solar masses and supermassive for masses in the range of $10^{6}$ to $10^{10}$ solar masses. But trying to elucidate the actual nature of the black hole from existing knowledge appears to be a very complex process for it involves a number of dilemmas such as the firewall paradox and Hawking radiation. Here, for simplicity, a probable nature of the black hole into 4D space-time and devoid of matter is presented based entirely on the MP model (Fig. 6) Rotation into forward time is defined by Kerr

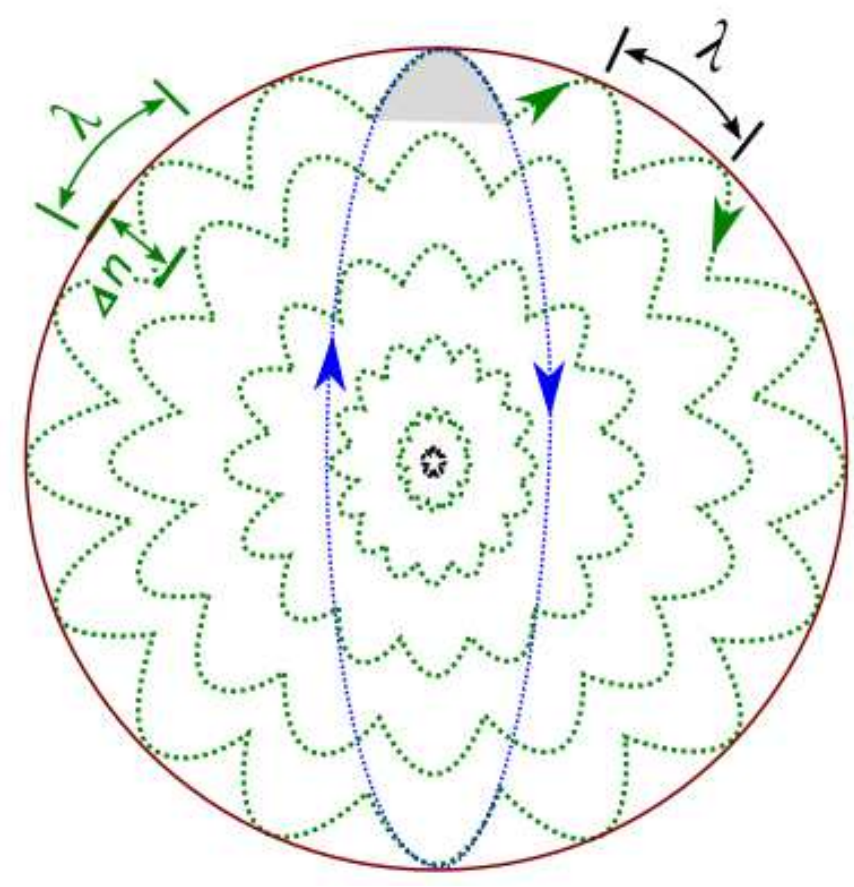

Figure 6. Application of the MP model to a black hole into 4D space-time and devoid of matter. The balance exerted by Einstein's gravity against forward time offers a static event horizon (maroon circle). Its interaction with light travelling in a straight path is expected to vanish along the orbital paths. This is progressively slowed into extra dimensions (green dotted loops) due to the orbital's precession into time reversal mode. Any decoherence (green wavelength) of minimal energy (gray area) is expected first to overcome coherent flow (black wavelength) beneath the event horizon. The outline of the MP field is given by the blue dotted shape for the simplest scenario (i.e., a hydrogen atom), while its multiples are comparable to a multielectron atom. Blue arrows = rotating MP field and green arrows = shift in precession into forward time. 
matrix, whereas the extra dimensions of inertia frames of BOs along the orbital paths are related to Schwarzschild property. The former is accorded to the precession of the MP field into forward time, while the latter becomes of extreme time dilation due to Einstein's gravity defined as circular acceleration or precession of the orbitals into time reversal mode (Fig. 4a and b). Any interaction with external light travelling in a straight path is intercepted by the orbital paths, while coherence is sustained at the event horizon. In the absence of matter, the emergence of the $\Psi$ and hence $\lambda$ towards observation is expected to be delayed. Thus, the transition of the $\Psi$ from point $x \rightarrow \hat{x}$ between two orbital paths becomes of gravitational time dilation and this can take light years to evolve especially at the macroscale (see also Fig. 5). In this case, whether any eventual outgoing radiation can account for gravitational wave types noted for a binary black hole merger [19] offers interesting prospects for further pursuits.

Comparably, a person falling into the black hole may never get the chance to reach singularity if one's body becomes elongated or 'spaghettized' along the orbital paths into extra dimensions of time infinite. Any outgoing information would probably be reflected in the same direction as the incoming light but this would be of time dilation due to gravity. If somehow Hawking radiation mimes $\pm h$ for the macroscale comparable to the microscale (Fig. $2 \mathrm{~d}$ ), its observation is limited to the emergence of the $\Psi$ described above. This possibly rules out the firewall paradox with some explanations on quantum entanglement provided in the preceding section. Applying such metaphors, the MP model's applicability to the solar system is examined next followed by a probable Big Bang scenario and cosmic inflation towards the end. 


\subsection{The solar system in a multiverse}

Based on the Nebular hypothesis, the solar system evolved from a cloud of dust and gases immediately after the Big Bang. Suppose the planetary bodies were formed within a UMF of the sun, a likely scenario is offered in Fig. 7. In this case, whether the stability of the solar system into space is sustained from interactions with others of similar type remains an open question not pursued here. Perhaps the solar system forms an MP field that is somewhat in thermal

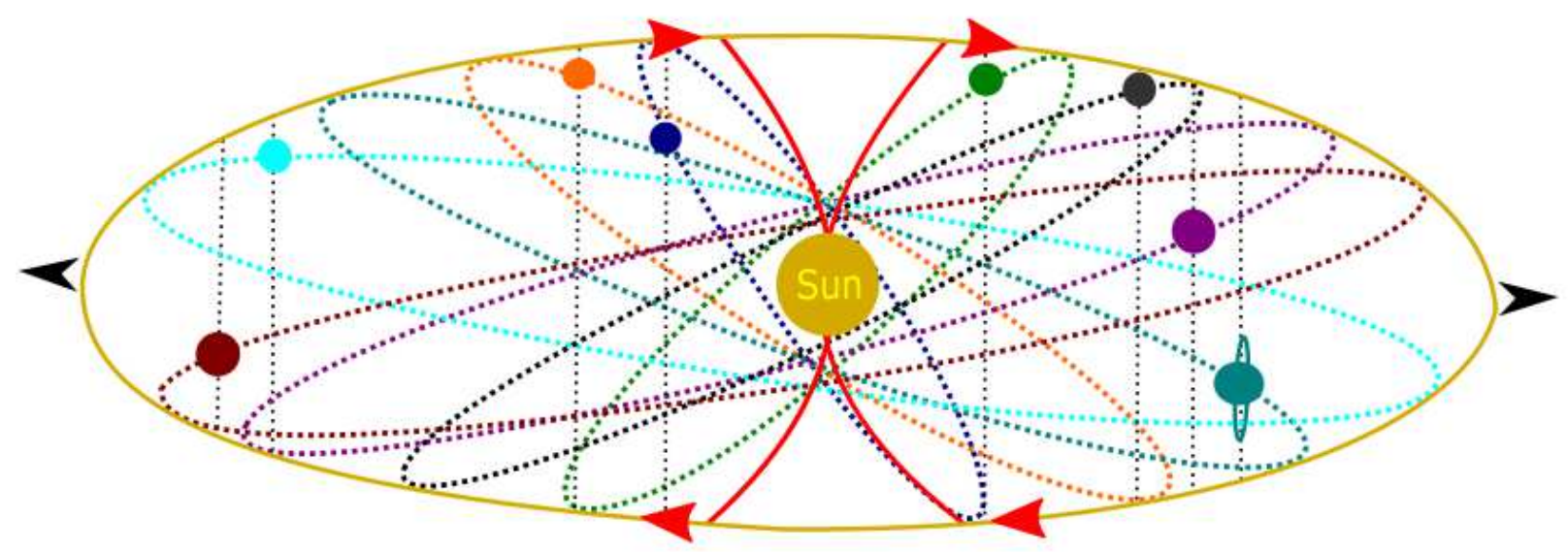

Figure 7. The application of the MP model to the solar system is comparable to a Rutherford planetary model into 4D space-time. The orbitals are quantized along straight paths of BOs (dotted lines). Divergence of the electromagnetic radiation from the sun (red curves and arrows) sustains $\vec{I}$ in asymmetry for the MP field (black arrows). The boundary (pale orange circle) indicates conservation. Note, the planets are not plotted according to size or type.

equilibrium within its galactic system known as the Milky Way. Such proposition remains a possibility because gravitational time dilation due to gravity would appear to spiral inwards from external light interactions - a scenario displayed by the Milky Way. Applying this intuition, the solar system is explored for the application of general relativity. 
In Fig. 8, an intuitive interpretation of Einstein field equation (EFE) of geometry [10] is applied with respect to the MP model. The scalar curvature, $R$ and the Ricci curvature tensor, $R_{u, v}$

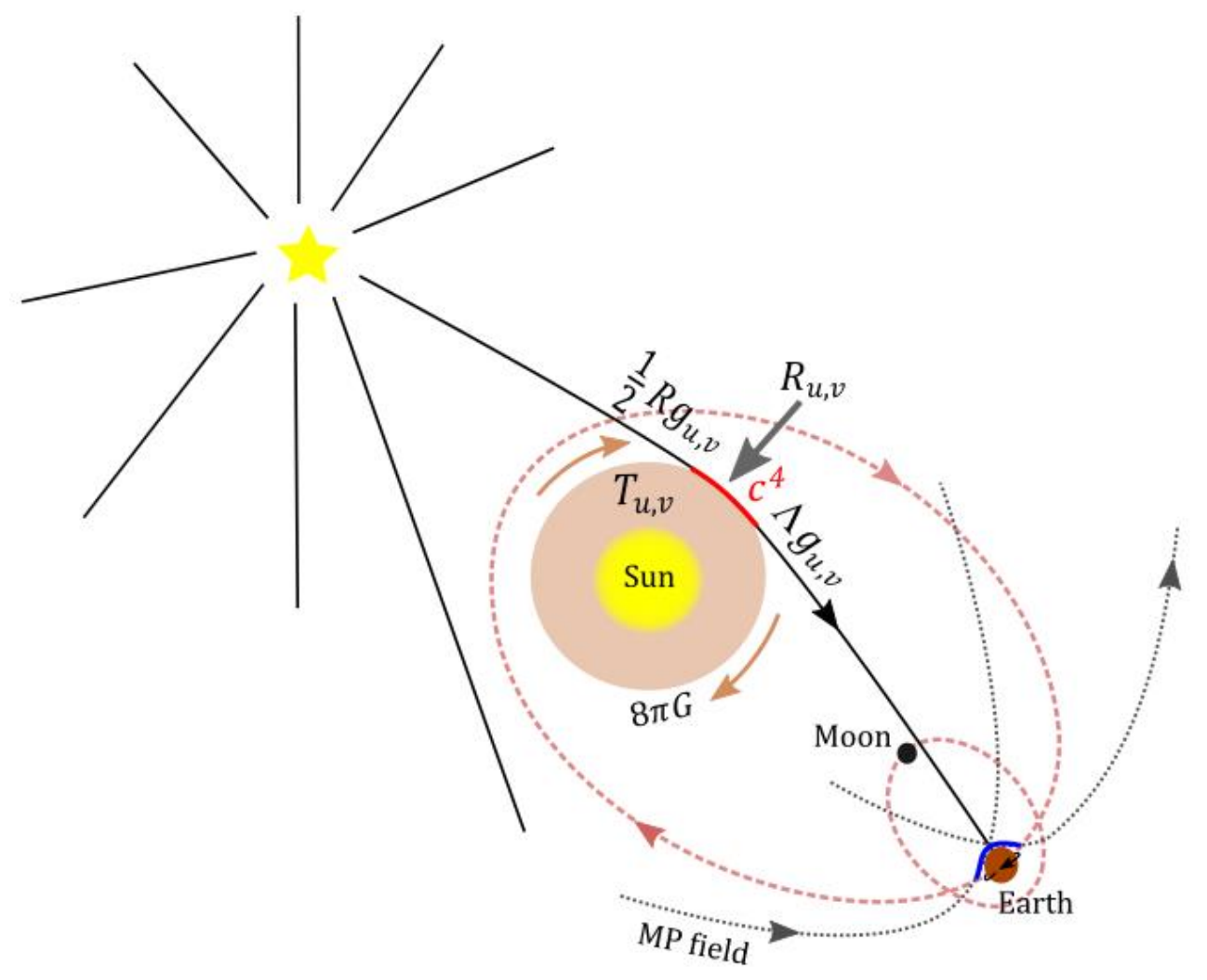

Figure 8. The application of the MP model to EFE of curved space-time. A light ray from a distant star on a straight path passes through the inertia frames of BOs. The path becomes bent by the sun's gravity in rotation and is redshifted with respect to an accelerated frame of reference on Earth (i.e., the equivalence principle). The sphere of the sun's gravitation field is localized but it determines how Earth should orbit into space-time against the precession of the MP field into forward time. The time evolution of the planetary $\Psi$ (blue curve) between two precessing MP fields is of time dilation due to gravity and this does not affect the incoming light path comparable to the microscale (e.g., Fig. 5). Note, the Earth's orbit and the MP field are not differentiated according to scale and this is expounded further in Fig. 9. 
describes the tensor field at the right angle of the light path interacting with the sphere of the rotating gravitational field. The path is bent at the starting point, where $x, y$ coordinates of spacetime is zero (i.e., $T_{0,0}$ ) for all reference frames of observation. The metric tensor, $g_{u, v}$ is applicable to both contraction, $\frac{1}{2} R g_{u, v}$ and expansion $\Lambda g_{u, v}$ of the light path. The stress-energy tensor, $T_{u, v}$ for the geodesic motion somewhat resemble inertia frames of the BO type into manifolds of extra dimensions (see also Fig. 2c). Its interaction with the light paths sustains relativistic features of $E=m c^{2}$ and the equivalence principle, while its curvature by gravity, $G$ induces, $g_{u, v}$. From these descriptions, a number of assumptions can be drawn.

1. Einstein's gravity is defined to be of a circular acceleration mode and is a consequence of precessing orbital paths into time reversal mode for a rotating body into space. This is a modified version of the equivalence principle and it is captured by Euler's formula (e.g., Fig. 5).

2. Gravity is localized within the MP and is applicable to a multiverse at a hierarchy of scales. Its domain of sphere determines the precession and spin of rotating smaller objects in orbit including curvature of light paths.

3. The $\Psi$ for classical objects takes a longer time frame to evolve due to extreme gravitational time dilation. Thus, it is considered negligibly small during observation at lightspeed and this sustains uniformity unlike the microscale.

These three assumptions are somewhat accommodated by the EFE and this is applicable to the development of the $\Psi$ at a hierarchy of scales. In Fig. 9, the $\Psi$ of a planetary body is shown by adapting Fig. 8. This considers that satellites orbit the planets in a similar fashion to how the planets orbit the sun and in turn electrons in orbit of the nucleus. Applying such intuitions, the plausible unification path for both the microscale and the macroscale is plotted next. 


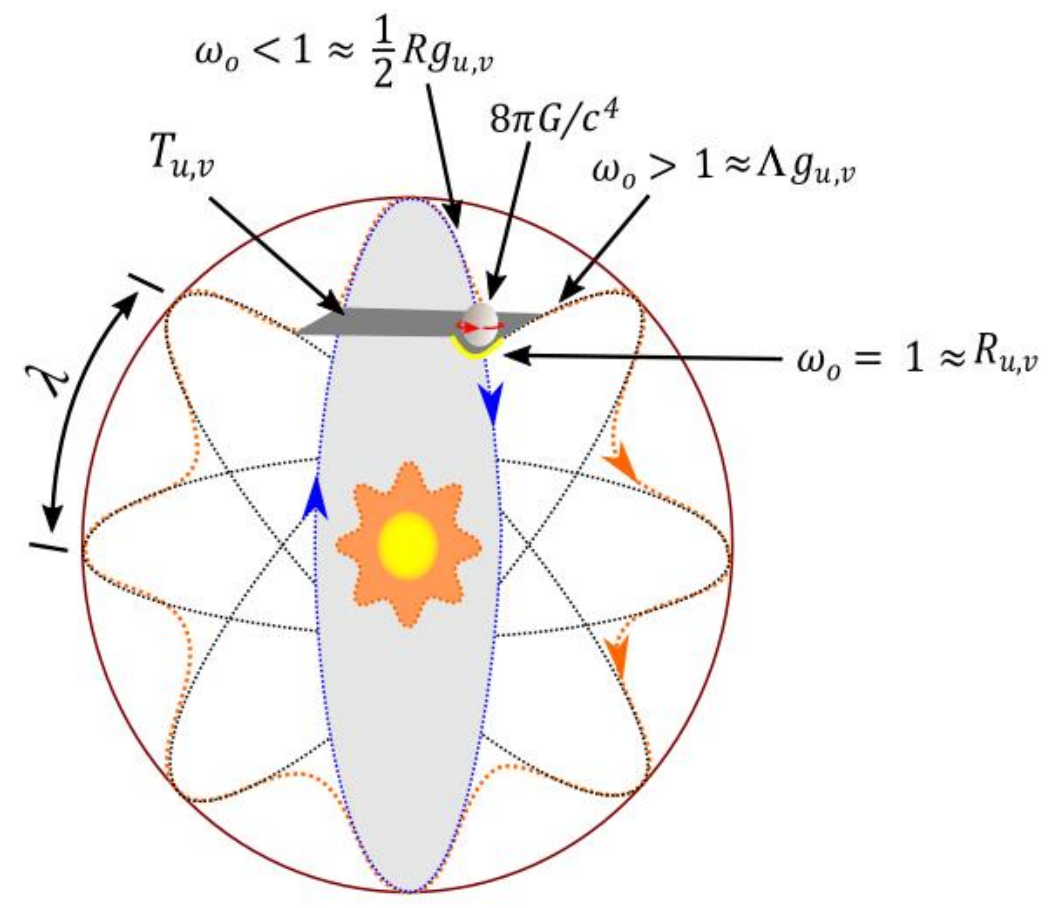

Figure 9. Application of EFE towards the generation of a planetary $\Psi$. The initiation of the $\Psi$ (yellow curve) is of gravitational time dilation and is comparable to the geodesic motion. Manifolds of $T_{u, v}$ into extra dimensions towards the sun mime the BOs (e.g., Fig. $2 b)$ and its curvature by gravity equates to $g_{u, v}$.

\subsection{A probable reconciliation path}

Pictorially, the MP model offers a tangible intuitive path towards the reconciliation process of quantum mechanics and general relativity based on the depiction of the $\Psi$ into 4D space-time (e.g., Fig. 5 and 9). Such a path is extremely difficult to plot using conventional methods either from bottom up such as string theories or top down like loop quantum gravity. For a crude mathematical representation, Equation 2 is expanded into the form

$$
i \int_{-\infty}^{\infty}(d R d T d g)_{u, v} \equiv i \int_{-\infty}^{\infty}(d \Omega d \phi d \theta)_{u, v}
$$


where $i$ refers to an object in orbit at an undefinable scale of an MP model in accordance with Euler's formulation (Fig. 5). Its momentum in both forward and reversible directions is of equal magnitude at the quantized states of BOs. From the geometry relationship of the proposed model, Equation 8 provides the link between the microscale and the macroscale in a multiverse. By expanding Equation 6, the planetary $\Psi$ is given by the expression

$$
8 \pi G T_{u, v} \int_{-\infty}^{\infty}\left(R+\frac{1}{2} R g+\Lambda g\right)^{2} d R_{u, v} \equiv n I_{z \|(u, v)} \int_{-\infty}^{\infty} \Psi^{*} \Psi d \tau_{u, v}
$$

The left side of Equation 9 relates the geodesic motion to the $\Psi$, where manifolds of the inertia frame of BO type in extra dimensions is given by $T_{u, v}$ (Fig. 9). The development of $\Psi$ is of gravitational time dilation, while its magnitude or the scale is defined by $8 \pi G$ with gravity localized within an MP field (e.g., Fig. 8). $R$ can be represented in 3D space-time $(u, v)$ and this somewhat mimes the 4D space-time. For Mercury, the perihelion precession of its elliptic orbit advances by 5,601 seconds of arc per century [13] for a body of a mass equal to $3.285 \mathrm{x} 10^{23} \mathrm{~kg}$. Suppose the formation of the arc equates to the approximate time it takes for the emergence of a planetary $\Psi$, this is attained at a rate of 56 seconds per year and is extremely time dilated due to Einstein's gravity. Thus, for observational purposes, a time machine is required to wind back the clock in order to register the complete $\Psi$ that evolved over the past centuries towards the present stage. Unfortunately, there is no such instrumentation that is capable of achieving a time travel feat either into the past or the future. 
With $\pm h$ projected outward towards the boundary of the MP field of time invariance, this sustains $\vec{I}$ in asymmetry (Fig. 2d). In this case, the uncertainty in the position of an object into space-time is defined by the relationship

$$
\left[8 \pi G T-\left(R+\frac{1}{2} R g+\Lambda g\right)\right]_{u, v} \cong(i \hbar-m c)_{u, v}
$$

Equation 10 is applicable at minimal energy level, where $\hbar$ is considered negligibly small and absorbed into the EFE for the macroscale. Any outgoing radiation towards the outer space in accordance with the $2^{\text {nd }}$ law of thermodynamics is attained at lightspeed for mass-energy equivalence, i.e., $-m c$. Alternatively, Equation 10 can be reduced into the form

$$
8 \pi G T_{u, v} \equiv i \hbar_{u, v}
$$

Equation 11 describes singularity, where Noether theorem of symmetry is applicable to the MP model into 4D space-time for a multiverse at a hierarchy of scales (see also section 3.0). The relativistic principles of $E=m c^{2}$ and the equivalence principle are sustained along BOs of the MP model into extra dimensions as mentioned earlier. Perhaps, for a multiverse within a visible universe, its outermost limit is defined by the cosmic microwave background (CMB) of an MP field type. How these all fit into a probable Big Bang scenario and cosmic inflation is presented next. 


\subsection{Other related cosmic themes}

Based on the alternative version of general relativity offered above, dark matter and dark energy are somewhat intuitively incorporated within the extra dimensions of the MP model within a multiverse of MP models at a hierarchy of scales. How the Big Bang and the accelerated cosmic inflation fit into this perspective is briefly expounded below in bullet points.

- The Big Bang scenario: Suppose the Big Bang evolved from a primordial soup in uniformity, its progression towards the lower hierarchy of scales ensues in the following manner: $S O(10) \rightarrow S U(5) \rightarrow S U(3)$ x $S U(2) \times U(1)$ and these curtailed forces of symmetries by assuming conservationism. The Lie group $S O(10)$ is a supersymmetry of multidimensional structures comparable to the $M$-theory type. To an extent, this physically mimes the topological surface area of the UMF background of a circular motion in flat space (Fig. 2b). Similarly, $S U(5)$ of the Lie group is accorded to the precessing MP field into forward time. It encompasses time reversal symmetry breaking of precessing orbitals into time reversal mode as mentioned earlier (Fig. 2d). The last term has three components and this is of the gauge symmetry for the atomic state [6]. $S U(3)$ is the strong nuclear force, $S U(2)$ is the weak nuclear force, while $U(1)$ represent electromagnetism. All these symmetries, together are somehow incorporated into the atomic MP model prior to light interaction. Based on Feynman path integral diagrams, the forces are mediated by bosons comparable to photons for electromagnetism. These are formed from coupling of particles and their antimatters at the fundamental level. So the photons as massless particles evolved first during the initial Big Bang state (Fig. 10a). Their inception process is comparable to reversal time symmetry breaking by Planck's radiation in accordance with the $2^{\text {nd }}$ law of thermodynamics for the microscale (Fig. $2 \mathrm{~d}$ ). 
a

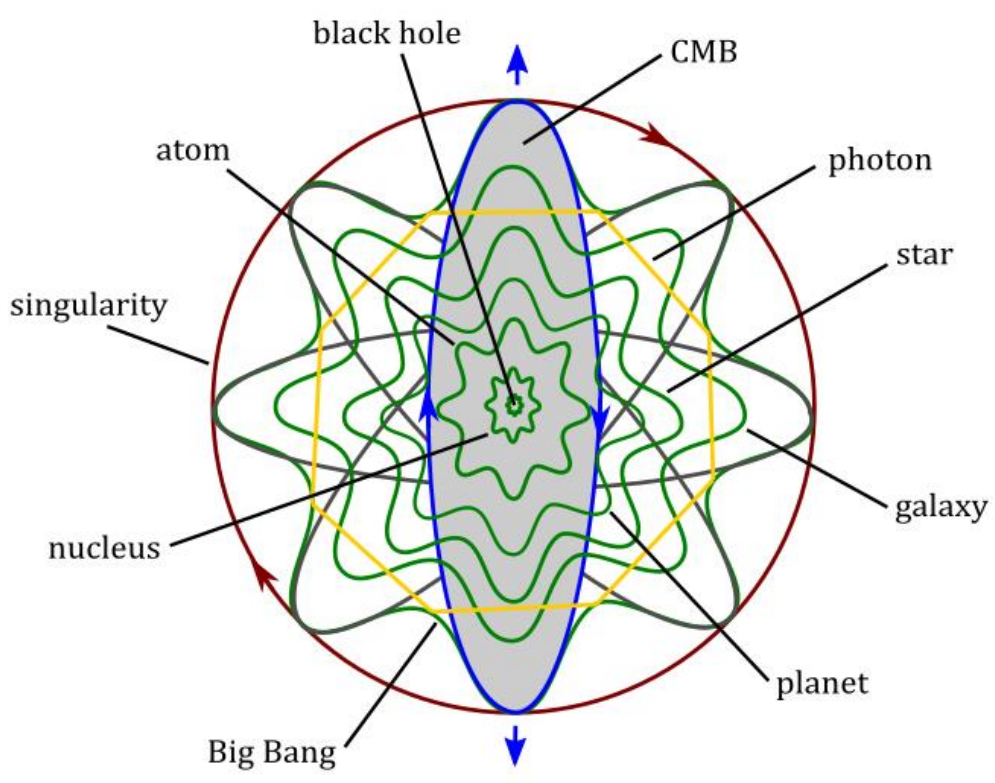

b

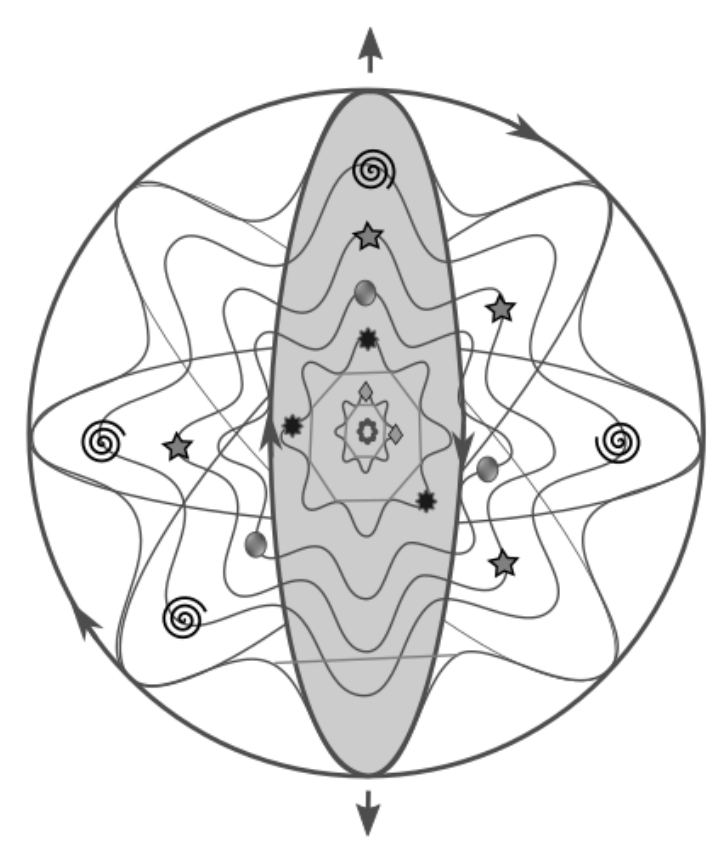

Figure 10. An idealized scenario of the Big Bang based on the MP model. (a) Time reversal symmetry due to Einstein's gravity is broken during the Big Bang from an initial state of uniformity or singularity. This insinuates the emergence of the MP field defined by the CMB. Cooling and regression towards the center produces multidimensional structures in thermal equilibrium. (b) Each subsequent dimension sets the stage for the evolution of bodies such as galaxy, star, planet and the atom and its constituents towards the minimal scale. A body evolves when matter collides and amalgamates into extra dimensions along the orbital paths in a multiverse. The emergence of MP models at any of the dimensions and devoid of matter insinuates a black hole such as at the centers of the galaxies and perhaps atoms.

For the visible universe defined by the CMB of an MP field, the photons assumed the inertia frames of BOs (Fig. 10a). Perhaps, the initial photons would have had a different nature and these gradually gained energy with interactions of BOs for a multiverse of MP models towards a lower hierarchy of scales (Fig. 10b). The descending order of interactions would follow; Big Bang $\rightarrow$ galaxies $\rightarrow$ stars $\rightarrow$ planets $\rightarrow$ atoms $\rightarrow$ nuclei 
and this is expected to generate various radiation types. In this case, whether the information gained by the photons is destroyed through quantum decoherence or conserved is open to further discussions. At the microscale, the atom is expected to store information about the Big Bang condition but concentrate energy comparable to the Higgs boson and gluon types. Perhaps, in the initial state, energies stored would have been sufficient to trigger both nuclear fusion and fission to generate various atom types and other exotic matters. In this case, the Big Bang assumes a low entropy state at a scale defined by the CMB (Fig. 10a). This is likened to a literal Wheeler-Feynman oneelectron-universe [21] comparable to the conceptualization process of the MP model (Fig. 1a, b, c and d). But how this evolve and by what process, including how matter and time originated and whether there exist other multiverses beyond the one defined by the CMB appear to be philosophical questions that are not explored in here. Only the applicability of the cosmic inflation to the MP model is offered next.

- A cosmic inflation scenario: Gravitational time dilation attained along BOs due to Einstein's gravity, severely impedes the emergence of the $\Psi$ so that the light paths are redshifted (e.g., Fig. 8). Depending on the scale, the time frames can be of light years for continual precession into forward time. To an internal observer in an inertia frame of reference, the shifted frequency, $v^{\prime}$ is defined by the generalized form, $v^{\prime}=v\left(1-\frac{G m}{r c^{2}}\right)$ into 2D flat space with $v$ is original frequency and $r$ is radius. For a localized universe of MP model precessing into forward time of a circular motion at the galactic scale, the redshift is considerably accelerated more than lightspeed according to Euler's formula (e.g., Fig. 5) to a distant observer on Earth (Fig. 10b). This can be described in the simplified form, $z=\frac{\lambda-\lambda_{0}}{\lambda_{0}} \propto d$ with $\lambda$ as the measured wavelength shift, $\lambda_{0}$ is the 
reference wavelength and $d$ is the measured distance. In this case, blueshifts are fairly constrained in accordance with the Hubble constant. Such explanations could perhaps account for why ordinary matter constitutes only $5 \%$ of the visible universe compared to $25 \%$ of dark matter and $70 \%$ of dark energy (e.g., Fig. 10b). Suppose dark matter is assumed by quantized states of BOs into extra dimensions of the MP models, this can somehow relate to weakly interacting massive particles [22] towards a higher hierarchy of scales in a multiverse. Dark energy is then assumed by interactions of light waves and the BOs into space comparable to $\Lambda$ application shown in Fig. 9. Such intuition offers one possible case why matter predominates over antimatter and this warrants further investigations.

- Further experimental pursuits: Comparable to the propositions offered for the microscale for further pursuits, a similar approach should be considered for the macroscale within the broad areas of cosmology and astrophysics. In this case, the external light beam is expected to be less than the area of the spinning object in orbit of a gyroscope for a number of scenarios. 1) Lightspeed at less than the gyroscope speed, 2) lightspeed equal to the gyroscope speed and 3) lightspeed at more than the gyroscope speed. Such undertakings should also consider the black hole scenario (Fig. 6) and general relativity (Fig. 8). Any successful outcomes are expected to ascertain the model's applicability to existing knowledge in physics for the macroscale as demonstrated in this section. 


\subsection{Concluding remarks}

Both quantum mechanics and general relativity are two extremely successful theories that accounts for observations made respectively for the microscale and the macroscale. However, their unification path continues to defy conventional methods for nearly a century despite the advancement of sophisticated instrumentation of our times. Suppose both theories are regarded to describe realities of nature at two extreme scales then the proposed MP model into 4D spacetime offers one tangible path towards their unification process. This considers a multiverse of MP models at a hierarchy of scales within the limits of the visible universe defined by the CMB. Einstein's gravity is then localized within the model and this is assumed to be of circular acceleration in time reversal mode against forward time. Symmetry breaking of time reversal insinuates the application of $2^{\text {nd }}$ law of thermodynamics. The relativistic features of $E=m c^{2}$ and the equivalence principle are sustained along the inertia frames of the model into extra dimensions. Such intuitions appear somewhat highly speculative, but the MP model is developed and applied in accordance with existing knowledge in physics and our general perceptions of the physical world. If considered, it offers a valuable intuitive tool that can complement well conventional methods, especially when attempting to integrate and consolidate many aspects of foundation physics into a proper perspective of 4D space-time for both the microscale and the macroscale. Such an undertaking may provide the needed incentives to explore physics further into the unknown realms using conventional methods, perhaps in incremental steps and this warrants further investigations. 


\section{Competing financial interests}

The author declares no competing financial interests.

\section{References}

1. T. J. Hollowood. Copenhagen quantum mechanics. Contemp. Phys. 57 (3), 289-308 (2016).

2. R. Kidd, J. Ardini and A. Anton. Evolution of the modern photon. Am. J. Phys. 57 (1), 27-35 (1989).

3. J. B. Hartle. Gravity: An introduction to Einstein's general relativity. (Addison-Wesley, San Francisco, 2003).

4. P. A. M. Dirac. Quantized singularities in the electromagnetic field. Proc. Roy. Soc. (London) A 133, 60 (1931).

5. J. P. Edwards and C. Schubert. Quantum mechanical path integrals in the first quantized approach to quantum field theory. arXiv preprint arXiv:1912.10004 (2019).

6. M. K. Gaillard, P. D. Grannis and F. J. Sciulli. The standard model of particle physics. Rev. Mod. Phys. 71 (2), S96 (1999).

7. J. Ellis. Outstanding questions: physics beyond the Standard Model. Phil. Trans. R. Soc. A 370, 818-830 (2012).

8. T. Rothman and S. Boughn. Can gravitons be detected?. Found. Phys. 36 (12), 18011825 (2006). 
9. Hooft, G. T. Graviton dominance in ultra-high-energy scattering. Phys. Lett. B 198 (1), 61-63 (1987).

10. P. de Aquino, K. Hagiwara, Q. Li and F. Maltoni. Simulating graviton production at hadron colliders. J. High Energy Phys. 2011 (6), 132 (2011).

11. R. Penrose. The road to reality: A complete guide to the laws of the universe. (Random House, Great Britain, 2005).

12. G. Amelino-Camelia. Quantum theory's last challenge. Nature 408 (6813), 661-664 (2000).

13. J. T. Cushing. Philosophical concepts in Physics: The historical relation between philosophy and scientific theories. (Cambridge University Press, United Kingdom, 1998).

14. C. D. Anderson. The positive electron. Phys. Rev. 43, 491 (1939).

15. H. Baer, V. Barger, M. Savoy and X. Tata. Multichannel assault on natural supersymmetry at the high luminosity LHC. Phys. Rev. D 94 (3), 035025 (2016).

16. A. G. Cornejo. A lagrangian solution for the precession of Mercury's perihelion. Int. J. Astron. 3.2, 31-34 (2014).

17. S. Weinberg. The trouble with quantum mechanics. New York Rev. Books 64 (1), 51-53 (2017).

18. N. White. Imaging black holes. Nature 407 (6801), 146-147 (2000).

19. B. P. Abbott et al. Observation of gravitational waves from a binary black hole merger. Phys. Rev. Lett. 116 (6), 061102 (2016).

20. R. Narayan and J. E. McClintock. Observational evidence for black holes. arXiv preprint arXiv:1312.6698 (2013). 
21. G. N. Ord and J. A. Gualtieri. The Feynman propagator from a single path. Phys. Rev. Lett. 89 (25), 250403 (2002).

22. S. Algeri, et al. Statistical challenges in the search for dark matter. arXiv preprint arXiv:1807.09273v1 (2018). 